\title{
Multiple Challenge Agent Different Category Indicator
}

National Cancer Institute

\section{Source}

National Cancer Institute. Multiple Challenge Agent Different Category Indicator. NCI

Thesaurus. Code C158299.

An indication as to whether the subject was challenged with more than one challenge agent from a different challenge agent category. 